\title{
EF-1 Peptide
}

National Cancer Institute

\section{Source}

National Cancer Institute. EF-1 Peptide. NCI Thesaurus. Code C49063.

A synthetic peptide derived from the protein ESW/FLI1 type 1 with potential use in cancer immunotherapy. EWS/FLI1 is a fusion protein frequently found in patients with Ewing's sarcoma or primitive neuroectodermal tumors (PNET). Vaccination with EF-1 peptide may stimulate the host immune response to elicit a cytotoxic $T$-cell response against tumor cells that express this EWS/FL1 type 1 fusion protein. 